\title{
Resonant excitation of trapped molecules in a molecular synchrotron
}

\author{
Peter C. Zieger, ${ }^{1,2}$ Chris J. Eyles, ${ }^{1}$ Gerard Meijer, ${ }^{1,3}$ and Hendrick L. Bethlem ${ }^{2}$ \\ ${ }^{1}$ Fritz-Haber-Institut der Max-Planck-Gesellschaft, Faradayweg 4-6, 14195 Berlin, Germany \\ ${ }^{2}$ LaserLaB, Department of Physics and Astronomy, VU University Amsterdam, De Boelelaan 1081, 1081 HV Amsterdam, The Netherlands \\ ${ }^{3}$ Institute for Molecules and Materials, Radboud University Nijmegen, Heijendaalseweg 135, 6525 AJ Nijmegen, The Netherlands
}

(Received 4 February 2013; published 26 April 2013)

\begin{abstract}
We characterize a synchrotron for polar molecules that consists of forty straight hexapoles arranged in a circle. By modulating either the voltages or the duration of the high-voltage pulses that are applied to the hexapoles, we shake the transverse and longitudinal well. If the frequency of the modulation matches a characteristic frequency of a stored molecule, the amplitude of the motion is resonantly excited, leading to a decrease in the number of molecules that are stored. From this, we determine the longitudinal, vertical, and radial frequencies that characterize the motion of the molecules inside the synchrotron and obtain knowledge about the couplings between the longitidinal and transverse motion. The measured frequencies are in good agreement with those obtained from three-dimensional trajectory calculations.
\end{abstract}

DOI: 10.1103/PhysRevA.87.043425

PACS number(s): 37.10.Pq, 37.10.Mn, 29.20.dk

\section{INTRODUCTION}

Resonant excitation is a process in which the amplitude of a system is increased under the influence of a small oscillatory force. It is a well-established technique for investigating and manipulating the motion of trapped ions and electrons [1-4] and is used on a regular basis to characterize the trap frequencies of magnetic and optical dipole traps of neutral atoms, including ring traps [5-9]. In this technique, the fields used for confinement are superimposed with a small external field. Particles whose motion is resonant with the frequency of this additional field experience a rapid increase in their oscillation amplitude until they are expelled from the trap. By measuring the number of trapped ions or atoms as a function of the frequency of the additional voltage, the characteristic motional frequencies of the trap can be mapped out.

In this paper, we use resonant excitation to study the motion of molecules inside a forty-piece molecular synchrotron. One of the goals of a molecular synchrotron [10] is to store multiple counterpropagating packets of molecules that interact with each other repeatedly. The collision signal at low energy gives detailed information that can be used as a benchmark for comparison with theoretical models for molecule-molecule interactions at an unprecedented level [11]. For these experiments, a full understanding of the motion of the molecules inside the synchrotron is essential. Here, we use resonant excitation to determine the longitudinal, vertical, and radial frequencies that characterize the motion of the molecules inside the synchrotron in a direct way and obtain knowledge about the couplings between the longitudinal and transverse motion. Due to the complexity of the electronics used for driving the synchrotron and the high voltages that are used, implementation of this technique is not straightforward. We present two different schemes to excite both the longitudinal and transverse motion of the molecules in the molecular synchrotron.

This paper is organized as follows. In Sec. II, we introduce a molecular synchrotron consisting of 40 straight hexapoles arranged in a circle and describe how the transverse and longitudinal well is shaken by modulating either the voltages or the duration of the high-voltage (HV) pulses that are applied to the hexapoles. In Sec. III, a mathematical model is presented that describes how a small external periodic force influences the motion of the molecules. Finally, in Sec. IV, the experimental results are shown and compared with results from a full three-dimensional (3D) simulation of the trajectories.

\section{EXPERIMENTAL SETUP}

A photograph of the molecular synchrotron is shown in the top panel of Fig. 1. The synchrotron is formed by placing 40 straight hexapoles on a circle with a diameter of $0.5 \mathrm{~m}$. The axes of the hexapoles make an angle of 9 deg with respect to each other. Each hexapole is composed of six cylindrical electrodes with a diameter of $4 \mathrm{~mm}$ and a nominal length of $37.4 \mathrm{~mm}$ that are held in place by a ceramic disk. The inner radius of each hexapole is $3.54 \mathrm{~mm}$. When a voltage difference of $6 \mathrm{kV}$ is applied between neighboring electrodes, the synchrotron is able to store deuterated ammonia molecules with a velocity between 110 and $140 \mathrm{~m} / \mathrm{s}$. These molecules are prepared by Stark deceleration of a pulsed molecular beam; details of the beamline are described elsewhere [12]. At the entrance of the synchrotron, the packet is several millimeters long and contains about a million molecules. All these molecules are in the upper inversion doublet component of a single rotational level (the $|J, M K\rangle=|1,-1\rangle$ level, i.e., the ground state of para-ammonia) in the vibrational and electronic ground state. Once the packet of molecules is inside the ring, the radially focusing hexapole fields are switched on, keeping the molecules on a circular orbit while continuously focusing them in the transverse direction. In the longitudinal directions, the packet is focused by temporarily increasing the voltages applied to the hexapoles to $8 \mathrm{kV}$ when the packet passes a gap between two adjacent hexapole sections [10].

The lower part of Fig. 1 shows a simplified schematic of the different types of oscillations performed by molecules inside the molecular synchrotron. The motion is best described by four characteristic frequencies. In keeping with the nomenclature used for charged particle accelerators, we refer to these as the cyclotron, synchrotron, and (horizontal 


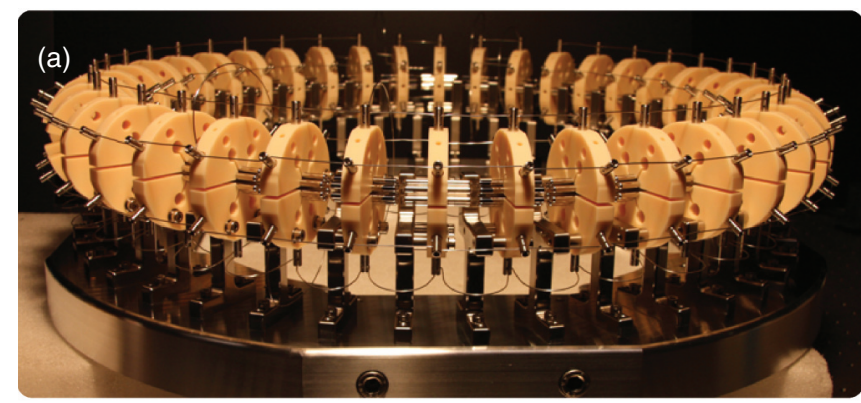

(b)

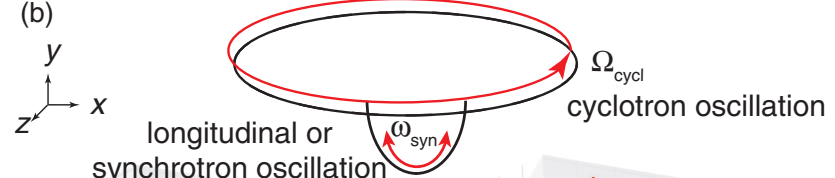
synchrotron oscillation

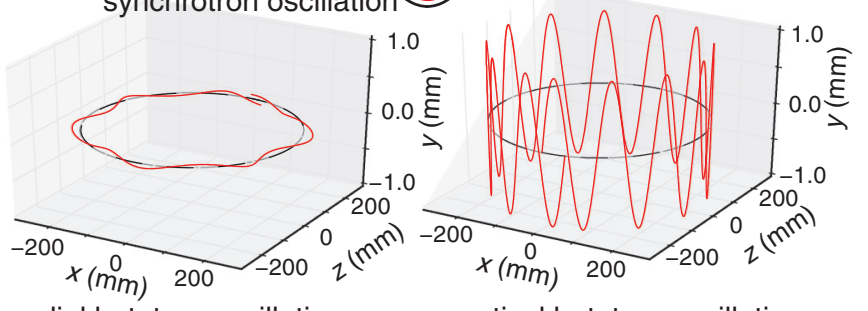

radial betatron oscillation $\omega_{r}$

vertical betatron oscillation $\omega_{y}$

FIG. 1. (Color online) (a) A photograph of the 40-piece molecular synchrotron. (b) A simplified schematic of the different types of oscillations performed by molecules inside the molecular synchrotron. The dashed black curves represent the trajectory of the synchronous molecule, the equilibrium orbit. The solid red (gray) curves show a trajectory of a molecule that oscillates around the synchronous molecule. The synchronous molecule revolves in the ring at the cyclotron frequency $\Omega_{\text {cycl }}$. The oscillation frequency of nonsynchronous molecules around the synchronous molecule in the longitudinal direction is called the synchrotron frequency $\omega_{\text {syn }}$. In the transverse directions the frequencies are called the radial betatron frequency, $\omega_{r}$, and the vertical betatron frequency, $\omega_{y}$.

and vertical) betatron frequencies. The dashed black curves represent the trajectory of a hypothetical molecule that makes a closed orbit in the synchrotron. We call this molecule the synchronous molecule, while we call its orbit the equilibrium orbit. By definition, the equilibrium orbit is at the position where the centrifugal force and the force created by the hexapoles cancel each other [13]. The cyclotron frequency is the inverse of the time that the molecule needs to complete one full round trip (round-trip time). The cyclotron frequency is $\Omega_{\text {cycl }}=2 \pi / t_{\mathrm{RT}}$. In our experiments $\Omega_{\text {cycl }} / 2 \pi$ is around $80 \mathrm{~Hz}$. While the cyclotron frequency describes the velocity of the traveling potential well, molecules will oscillate in all three directions within this moving frame. In the longitudinal direction the molecules oscillate at the synchrotron frequency, $\omega_{\text {syn }}$. In the transverse directions the molecules oscillate with the horizontal and vertical betatron frequency, $\omega_{r}$ and $\omega_{y}$, respectively. The characteristic frequencies depend on the confining voltages that are applied on the electrodes as well as on the velocity of the traveling potential well.

Figure 2 shows the density of ammonia molecules as a function of time after injection into the synchrotron for a series of selected numbers of round trips. In this measurement 13 packets are injected, after which the loading is stopped. These

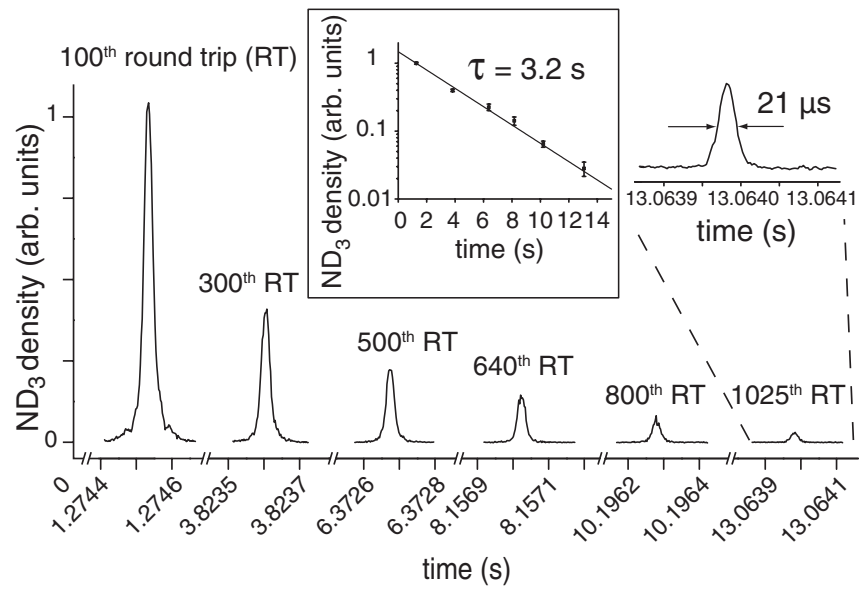

FIG. 2. Measurements of the density of $\mathrm{ND}_{3}$ molecules as a function of time (in seconds) for a selected number of round trips. The observed temporal width of $21 \mu$ s after 1025 round trips corresponds to a packet length of $2.6 \mathrm{~mm}$ after a flight distance of more than a mile. The inset shows the exponential decay of the signal with time.

packets trail each other by a distance of three hexapoles. The first and the last packet are four hexapoles apart. The different peaks correspond to a characteristic number of round trips; e.g., for the 640th round trip the molecular packet was confined for $8.15 \mathrm{~s}$, corresponding to a flight length of $1 \mathrm{~km}$. Even after 1025 round trips, i.e., after the molecules have traveled further than a mile and have passed through a gap 41,000 times, their signal can be clearly recognized. The temporal width of $21 \mu$ s corresponds to a packet length of $2.6 \mathrm{~mm}$. This measurement explicitly demonstrates the stability of the trajectories of molecules inside a molecular synchrotron. The density of ammonia molecules is seen to decay exponentially with time at a rate of $0.31 \mathrm{~s}^{-1}$ (see inset of Fig. 2). This is the lowest decay rate that has been observed for neutral ammonia molecules in any trap to date.

In this paper, we study the motion of molecules inside the molecular synchrotron using the resonant excitation technique. Resonant excitation relies on the fact that a periodic small deviation with a frequency that is close to a characteristic frequency increases the amplitude of the motion of the molecules inside the molecular synchrotron. This shaking of the potential well can be realized in two different ways, as sketched in Fig. 3. To excite the longitudinal motion, the time duration of the bunching pulse $\Delta t_{\text {bunch }}$ is prolonged or reduced at a frequency $\omega_{\text {ext }}$. In our experiments, the bunching pulse typically has a duration of $40 \mu s$ and is prolonged or reduced by $2 \mu \mathrm{s}$. To excite the transverse motion, the voltages applied to the negative electrodes are modulated. In our experiments, this voltage is typically $-4 \mathrm{kV}$ and is modulated by a sinusoidal voltage with an amplitude of $20 \mathrm{~V}$.

\section{ANALYTICAL MODEL}

Before discussing the experimental results, we first present an analytical model that describes the effect of the excitation on the motion of the molecules. Molecules that are trapped inside the synchrotron will oscillate radially, vertically, and longitudinally around the synchronous molecule. Let us first consider the motion in the radial direction. With no external 
(a) Longitudinal excitation

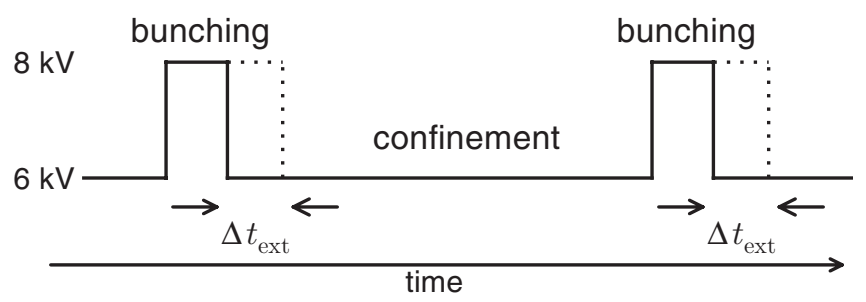

(b) Transverse excitation

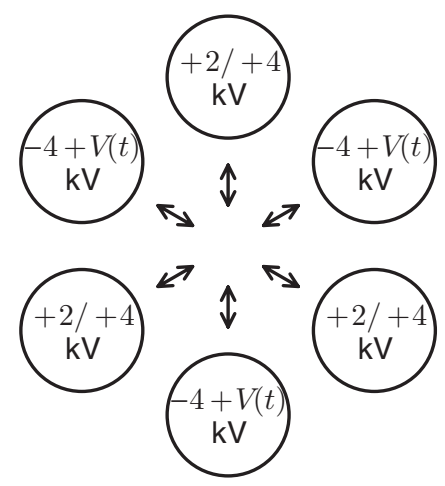

FIG. 3. Different schemes to excite the longitudinal and transverse motions of the molecules in the molecular synchrotron. (a) Excitation of the longitudinal motion. The time duration of the bunching pulse $\Delta t_{\text {bunch }}$ in the switching scheme is prolonged or reduced by $\Delta t_{\text {ext }}$ at frequency $\omega_{\text {ext }}$. (b) Excitation of the transverse motion. As indicated by the arrows, the magnitude of the electric field varies only in the transverse direction.

ac field present, the time-dependent radial position $r(t)$ can be written, to a first approximation, as

$$
r(t)=r_{\text {equi }}+r_{0} \cos \left(\omega_{r} t\right),
$$

in which $r_{\text {equi }}$ is the position of the synchronous molecule with respect to the geometrical center of the hexapole, $r_{0}$ is the amplitude of the oscillation, and $\omega_{r}$ is the radial betatron oscillation frequency. The corresponding radial velocity $v_{r}(t)$ is

$$
v_{r}(t)=-r_{0} \omega_{r} \sin \left(\omega_{r} t\right) .
$$

If the electric field is now modulated by an external frequency $\omega_{\text {ext }}$, the molecule experiences a time-dependent force $f(r, t)$ which can be approximated by

$$
f(r, t)=-k_{0}\left[1+\delta \sin \left(\omega_{\mathrm{ext}} t\right)\right] r(t) .
$$

Here, $k_{0}$ is the force constant of the hexapole field and $\delta$ describes the amplitude of the external oscillation. In our experiments, $\delta$ is below $1 \%$. We are now interested in the energy that is transferred from the external source to the motion of the molecules, i.e., the work done on the molecule:

$$
\begin{aligned}
W_{r}(t) & =\int_{0}^{r(t)} f(r, t) d r=\int_{0}^{t} f(r, t) \frac{d r}{d t} d t \\
& =\int_{0}^{t} f(r, t) v_{r}(t) d t .
\end{aligned}
$$

Equations (1), (2), (3), and (4) can now be combined to give

$$
\begin{aligned}
W_{r}(t)= & \delta \int_{0}^{t}\left[-k_{0} \sin \left(\omega_{\text {ext }} t\right)\right] \\
& \times\left[r_{\text {equi }}+r_{0} \cos \left(\omega_{r} t\right)\right]\left[-r_{0} \omega_{r} \sin \left(\omega_{r} t\right)\right] d t \\
= & \delta \omega_{r} k_{0} r_{\text {equi }} \int_{0}^{t} r_{0} \sin \left(\omega_{r} t\right) \sin \left(\omega_{\text {ext }} t\right) d t \\
& +\delta \omega_{r} k_{0} \int_{0}^{t} r_{0}^{2} \sin \left(\omega_{r} t\right) \cos \left(\omega_{r} t\right) \sin \left(\omega_{\text {ext }} t\right) d t,
\end{aligned}
$$

where we have neglected the part in Eq. (3) that does not depend on the external frequency as it will average to zero. Using elementary algebra, Eq. (5) can be simplified to

$$
\begin{aligned}
W_{r}(t)= & \delta \omega_{r} k_{0} r_{\text {equi }} \int_{0}^{t} \frac{r_{0}}{2}\left[\cos \left(\left(\omega_{r}-\omega_{\text {ext }}\right) t\right)\right. \\
& \left.-\cos \left(\left(\omega_{r}+\omega_{\text {ext }}\right) t\right)\right] d t \\
& +\delta \omega_{r} k_{0} \int_{0}^{t}\left(\frac{r_{0}}{2}\right)^{2}\left[\cos \left(\left(2 \omega_{r}-\omega_{\text {ext }}\right) t\right)\right. \\
& \left.-\cos \left(\left(2 \omega_{r}+\omega_{\text {ext }}\right) t\right)\right] d t .
\end{aligned}
$$

The integrals in Eq. (6) become zero unless $\omega_{\text {ext }}=\omega_{r}$ (first integral) or $\omega_{\mathrm{ext}}=2 \omega_{r}$ (second integral). Equation (6) thus predicts resonances at the fundamental and the second harmonic of the radial betatron frequency. In principle, also the strength of the resonances should follow from this model, but as $r_{0}$ is no longer constant (since the magnitude of the oscillation increases as it is driven) an analytical solution cannot be found.

The same derivation and argumentation can be used to describe the motion in the vertical and longitudinal directions. In the vertical direction, the equilibrium position is at $y=0$ (neglecting gravity) and a resonance is expected only at $2 \omega_{y}$. In the longitudinal direction, the motion is excited by asymmetrically prolonging and reducing the duration of the bunching pulse as sketched in the upper panel of Fig. 3. This implies that both the depth of the moving potential and its speed are modulated. As a result, resonances are expected at $\omega_{\text {syn }}$ and $2 \omega_{\text {syn }}$.

In order to derive Eq. (6), we assumed that the motions in the three directions are completely uncoupled. In this case only resonances are expected at $\omega_{r}, 2 \omega_{r}, 2 \omega_{y}, \omega_{\text {syn }}$, and $2 \omega_{\text {syn }}$. In the synchrotron, however, the different motions are strongly coupled in several ways. For instance, the radial motion is coupled strongly to the longitudinal motion due to virtual (Coriolis) forces that act in the rotating frame. Furthermore, the radial motion is coupled to the vertical motion due to the inversion splitting and due to the fact that the electrodes are round rather than hyperbolic [13]. Lastly, the radial and vertical confinement forces are a function of the position within a hexapole; i.e., towards the gaps the forces are smaller than in the middle of the hexapole. As a result of these different couplings the amplitude of the motion in one direction is modulated at the trapping frequencies in the other directions. For the amplitude of the radial motion, expressed in Eq. (1), this implies, for instance, that it now contains an additional term that depends on the synchrotron frequency:

$$
r(t)=\left[1+\epsilon \sin \left(\omega_{\text {syn }} t+\phi\right)\right]\left[r_{\text {equi }}+r_{0} \cos \left(\omega_{r} t\right)\right],
$$


with $\epsilon$ being a number smaller than 1 that represents the coupling between the radial and synchrotron motion. If we use Eq. (7) and again calculate the work that is done on the molecule by the external force, we now find not only resonance frequencies at $\omega_{r}$ and $2 \omega_{r}$, but also at $\omega_{r}+\omega_{\text {syn }}$ and $\omega_{r}-\omega_{\text {syn }}$, and similarly for the other motions.

\section{EXPERIMENTAL RESULTS}

Figure 4 shows typical excitation measurements. In these measurements, 13 packets of $\mathrm{ND}_{3}$ molecules with a forward velocity of $124.3 \mathrm{~m} / \mathrm{s}$ make 100 round trips within the synchrotron before being detected by a pulsed uv laser. The signal is averaged over 50 shots. The black curves shown in the top and bottom panels are the data obtained when the motion is excited using the scheme shown in the top and bottom panels of Fig. 3, respectively. As expected from the equations deduced in Sec. III, the signal is only affected when the driving frequency is resonant with one of the characteristic frequencies in the synchrotron or a combination thereof. The observed resonances, together with their assignment, are listed in Table I. The gray curves shown below the measurements in Fig. 4 result from 3D simulations using the electric field calculated by SIMION [14] and the experimental settings. In these simulations, the trajectories of 3000 molecules

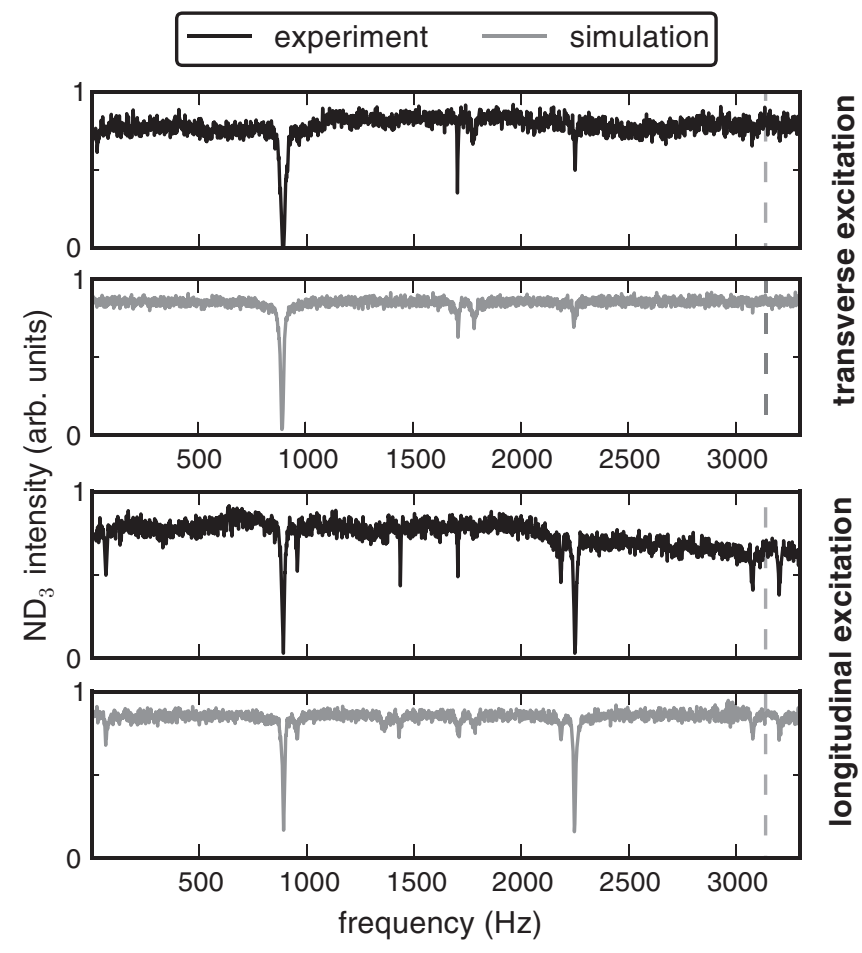

FIG. 4. The top panel shows the $\mathrm{ND}_{3}$ intensity as a function of the frequency at which the transverse potential is modulated (black curve). Underneath the simulated spectrum is shown in gray. The dashed gray vertical line indicates the frequency $40 \Omega_{\text {cycl }} / 2 \pi=$ $3138 \mathrm{~Hz}$. For the transverse frequency scan the assignment of the resonances is shown in the left side of Table I. The lower panel shows the excitation measurement in the longitudinal direction (black curve) together with the simulated spectra. For the experimental longitudinal frequency scan the assignment of the resonances is shown in the right side of Table I.
TABLE I. The detected resonances (in hertz) from Fig. 4 together with their assigned angular frequencies for the transverse and longitudinal frequency scans.

\begin{tabular}{lcccc}
\hline \hline \multicolumn{2}{c}{ Transverse measurement } & & \multicolumn{2}{c}{ Longitudinal measurement } \\
\cline { 1 - 1 } $\begin{array}{l}\text { Frequency } \\
(\omega / 2 \pi)\end{array}$ & Assignment & & $\begin{array}{c}\text { Frequency } \\
(\omega / 2 \pi)\end{array}$ & Assignment \\
\hline & & $64 \mathrm{~Hz}$ & $\omega_{\text {syn }}$ \\
$890 \mathrm{~Hz}$ & $\omega_{r}$ & & $890 \mathrm{~Hz}$ & $\omega_{r}$ \\
& & $955 \mathrm{~Hz}$ & $\omega_{r}+\omega_{\text {syn }}$ \\
& & $1437 \mathrm{~Hz}$ & $40 \Omega_{\text {cycl }}-2 \omega_{y}$ \\
$1701 \mathrm{~Hz}$ & $2 \omega_{y}$ & $1704 \mathrm{~Hz}$ & $2 \omega_{y}$ \\
$1780 \mathrm{~Hz}$ & $2 \omega_{r}$ & & \\
& & $2184 \mathrm{~Hz}$ & $40 \Omega_{\text {cycl }}-\left(\omega_{r}+\omega_{\text {syn }}\right)$ \\
$2251 \mathrm{~Hz}$ & $40 \Omega_{\text {cycl }}-\omega_{r}$ & $2250 \mathrm{~Hz}$ & $40 \Omega_{\text {cycl }}-\omega_{r}$ \\
& & $3075 \mathrm{~Hz}$ & $40 \Omega_{\text {cycl }}-\omega_{\text {syn }}$ \\
& & $3204 \mathrm{~Hz}$ & $40 \Omega_{\text {cycl }}+\omega_{\text {syn }}$ \\
\hline \hline
\end{tabular}

were calculated at each excitation frequency. If an excited molecule is expelled from the synchrotron, we store along which coordinate the molecule exits the synchrotron. This information is used to check the assignment of the different resonances.

Let us first focus on the transverse frequency scan. Here, four resonances are detected. The strongest depletion is at $890 \mathrm{~Hz}$ and corresponds to the characteristic radial frequency. No depletion of signal is found at the fundamental vertical frequency of $850 \mathrm{~Hz}$ but a relatively strong resonance is observed at $1701 \mathrm{~Hz}$, which corresponds to the second harmonic in the vertical direction. The peak next to it (at $1780 \mathrm{~Hz}$ ) is much weaker and results from the second harmonic of the radial frequency. The last peak at $2251 \mathrm{~Hz}$ results from a coupling of the radial frequency with the cyclotron frequency and is interpreted as $\left(40 \Omega_{\mathrm{cycl}}-\omega_{r}\right) / 2 \pi$.

The simulated spectrum shows all the expected characteristic resonances. Only for the second harmonic of the vertical resonance is the experiment much stronger than in the simulation. As mentioned before, the strength of the second harmonic resonance compared to the fundamental is influenced by the spatial width of the molecular packet. We attribute this to the fact that the simulation does not perfectly reproduce the experimental phase space distribution.

The longitudinal excitation is shown in the lower panel of Fig. 4. Compared to the excitation in the transverse direction, this experimental frequency spectrum shows most of the already observed resonances and some new ones. The extra resonances occur primarily due to the coupling of the synchrotron and cyclotron motion to the other motions. The fundamental synchrotron frequency is observed at $65 \mathrm{~Hz}$. Its second harmonic frequency is not visible. The depletion observed at $955 \mathrm{~Hz}$ is due to the coupling of the synchrotron motion with the radial frequency. A resonance is observed at a frequency of $\left(\omega_{r}+\omega_{\text {syn }}\right) / 2 \pi$ but not at a frequency of $\left(\omega_{r}-\omega_{\text {syn }}\right) / 2 \pi$, in agreement with the simulations. We attribute this asymmetric coupling to peculiarities in the formulas describing the Coriolis force. A more exact model for this phenomena is currently lacking. The modulation of the confinement force due to the 40 -fold symmetry of the ring gives rise to a number of resonances; for instance, the 


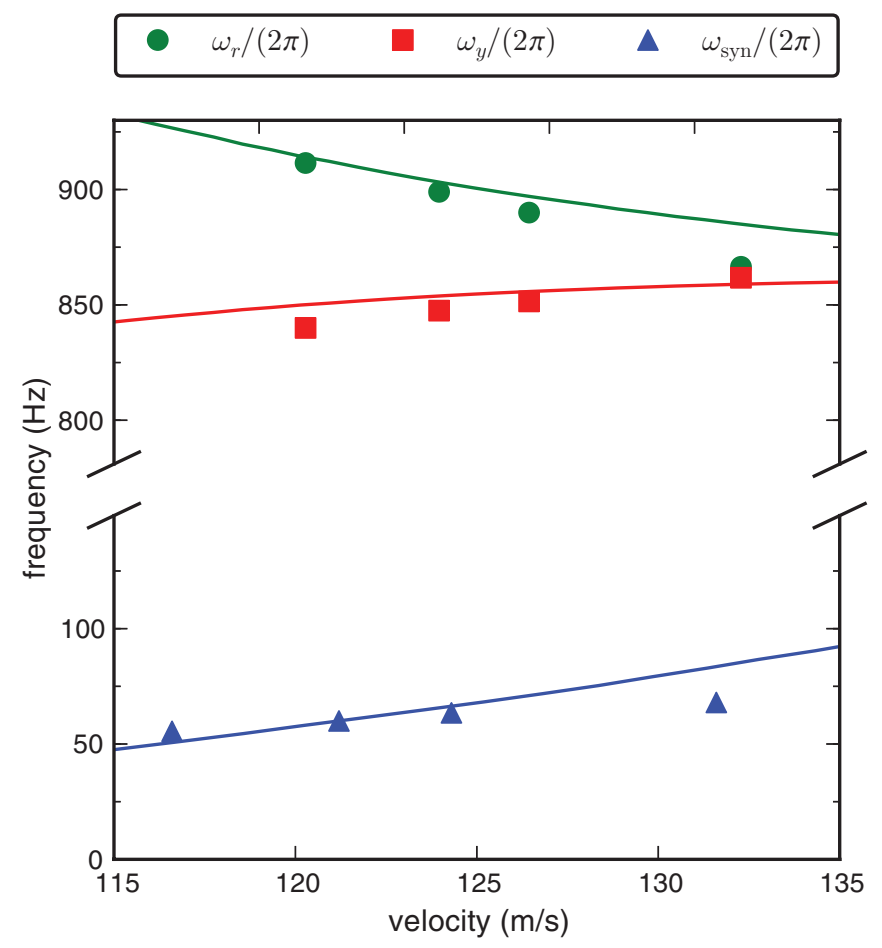

FIG. 5. (Color online) The characteristic trap frequencies as a function of the velocity of the molecular packet. The circles, squares, and triangles correspond to the longitudinal, vertical, and radial frequencies, respectively, derived from excitation measurements performed at different velocities. The theoretical velocity-dependent frequencies are drawn as solid lines.

resonance at $3075 \mathrm{~Hz}$, which is interpreted as $\left(40 \Omega_{\mathrm{cycl}}-\right.$ $\left.\omega_{\text {syn }}\right) / 2 \pi$.
When, at a given voltage, the velocity of the injected packet is varied, the equilibrium orbit of the molecular packet is altered. This changes all three characteristic frequencies accordingly. The results of such experimental measurements are shown in Fig. 5. Note that at a constant voltage, the trajectories are only stable at specific velocities (see Ref. [15]). The radial, vertical, and longitudinal characteristic frequencies are represented by circles, squares, and triangles, respectively. The solid lines in Fig. 5 show the theoretically predicted trap frequencies as function of velocity found by analyzing the electric field around the equilibrium orbit. For the longitudinal frequencies all three spatial dimensions were considered. For the radial and vertical directions a two-dimensional model was used.

\section{CONCLUSION}

In this paper we have shown that resonance excitation is a powerful tool to characterize the motion of polar molecules inside a molecular synchrotron. The measured characteristic frequencies are in good agreement with those found from a 3D model, which shows that we have a good understanding of the trajectories. This is essential for future applications of the synchrotron.

\section{ACKNOWLEDGMENTS}

We acknowledge the electronics design by G. Heyne, V. Platschkowski, and T. Vetter, the mechanical design by A. J. A. van Roij, and the software support by U. Hoppe. C.J.E. thanks the Alexander von Humboldt Foundation for a postdoctoral fellowship. G.M. acknowledges support from the ERC-2009-AdG under Grant Agreement 247142-MolChip. H.L.B. acknowledges financial support from NWO via a VIDI grant and from the ERC via a starting grant.
[1] L. S. Brown and G. Gabrielse, Rev. Mod. Phys. 58, 233 (1986).

[2] H. Dehmelt, Rev. Mod. Phys. 62, 525 (1990).

[3] W. Paul, Rev. Mod. Phys. 62, 531 (1990).

[4] R. E. March, Mass Spectr. Rev. 28, 961 (2009).

[5] J. A. Sauer, M. D. Barrett, and M. S. Chapman, Phys. Rev. Lett. 87, 270401 (2001).

[6] S. Wu, W. Rooijakkers, P. Striehl, and M. Prentiss, Phys. Rev. A 70, 013409 (2004).

[7] S. Gupta, K. W. Murch, K. L. Moore, T. P. Purdy, and D. M. Stamper-Kurn, Phys. Rev. Lett. 95, 143201 (2005).

[8] A. S. Arnold, C. S. Garvie, and E. Riis, Phys. Rev. A 73, 041606(R) (2006).

[9] A. Ramanathan, K. C. Wright, S. R. Muniz, M. Zelan,
W. T. Hill, C. J. Lobb, K. Helmerson, W. D. Phillips, and G. K. Campbell, Phys. Rev. Lett. 106, 130401 (2011).

[10] P. C. Zieger, S. Y. T. van de Meerakker, C. E. Heiner, H. L. Bethlem, A. J. A. van Roij, and G. Meijer, Phys. Rev. Lett. 105, 173001 (2010).

[11] S. Y. T. van de Meerakker, H. L. Bethlem, and G. Meijer, Nat. Phys. 4, 595 (2008).

[12] C. E. Heiner, H. L. Bethlem, and G. Meijer, Phys. Chem. Chem. Phys. 8, 2666 (2006)

[13] F. M. H. Crompvoets, H. L. Bethlem, and G. Meijer, Adv. Atom., Mol. Opt. Phys. 52, 209 (2005).

[14] D. A. Dahl, SIMION 3D version 8.0, 1995, www.simion.com.

[15] C. E. Heiner, G. Meijer, and H. L. Bethlem, Phys. Rev. A 78, 030702 (2008). 